\title{
EchoGéo
}

$32 \mid 2015$

Inde : le grand écart spatial

\section{Entre tensions et souplesse : le grand écart spatial dans la diffusion de l'enseignement préscolaire au Tamil Nadu (Inde du sud)}

\section{Émilie Ponceaud-Goreau}

\section{CpenEdition}

Journals

Édition électronique

URL : https://journals.openedition.org/echogeo/14268

DOI : $10.4000 /$ echogeo. 14268

ISSN : 1963-1197

Éditeur

Pôle de recherche pour l'organisation et la diffusion de l'information géographique (CNRS UMR 8586)

Référence électronique

Émilie Ponceaud-Goreau, «Entre tensions et souplesse : le grand écart spatial dans la diffusion de l'enseignement préscolaire au Tamil Nadu (Inde du sud) », EchoGéo [En ligne], 32 | 2015, mis en ligne le 15 juillet 2015, consulté le 10 août 2021. URL : http://journals.openedition.org/echogeo/14268 ; DOI : https://doi.org/10.4000/echogeo.14268

Ce document a été généré automatiquement le 10 août 2021.

EchoGéo est mis à disposition selon les termes de la licence Creative Commons Attribution - Pas d'Utilisation Commerciale - Pas de Modification 4.0 International (CC BY-NC-ND) 


\title{
Entre tensions et souplesse : le grand écart spatial dans la diffusion de l'enseignement préscolaire au Tamil Nadu (Inde du sud)
}

\author{
Émilie Ponceaud-Goreau
}

\section{Introduction}

1 L'importance des relations entre éducation et développement (Buisson, 2009, p. 19) a largement été affirmée et diffusée par l'Unesco et les grands bailleurs de fonds internationaux qui encouragent aujourd'hui la préscolarisation des enfants de 3 à 6 ans. Depuis les années 2000 où l'éducation et la protection de la petite enfance sont devenues un des objectifs du Millénaire, ils l'envisagent comme un investissement en termes de capital humain et insistent sur l'importance de commencer la scolarisation au plus tôt afin de compenser un certain nombre d'inégalités, d'améliorer le bien-être et la scolarisation future de l'enfant (Rapport Unesco, 2007, p. 4). En Inde, l'impulsion de l'État a été primordiale et s'est traduite par des politiques publiques actives. La préscolarisation est présente dès 1975 dans les anganwadis qui accueillent les enfants de moins de six ans et procurent des services de santé ou de distribution de nourriture et de pensions pour les personnes les plus fragiles, sur l'ensemble du territoire. Les populations les plus modestes étaient marquées par une politique autoritaire de contrôle des naissances, dans un contexte de malnutrition, de forte mortalité infantile, de très faible inscription à l'école et de fort décrochage scolaire (Gupta, 2012, p. 246-248). La préscolarisation, associée à la lutte contre la malnutrition et les vaccinations, était ainsi un levier au développement pour une population cible.

2 L'Inde atteindra en 2015 l'objectif fixé par l'Unesco d'un taux brut de scolarisation dans l'enseignement préprimaire de $80 \%$ (Rapport Unesco, 2014, p. 50). La fréquentation d'une $_{\text {preschool }}^{1} \mathrm{y}$ est rapidement devenue une norme, une condition de réussite 
scolaire, et l'entrée à l'école primaire ${ }^{2}$ du quartier ne marque plus le début de la scolarisation. Sous l'influence de la mondialisation, les pratiques éducatives familiales se sont homogénéisées et la préscolarisation est aujourd'hui associée à la modernité. Simultanément, la libéralisation de l'économie indienne depuis 1991 a permis l'explosion du secteur privé. L'éducation n'échappe pas à ce processus. Les familles les plus aisées se sont directement adressées au secteur privé particulièrement réactif et à l'offre abondante dans le champ de l'éducation préscolaire. S'ajoutant à un secteur public jusque-là focalisé sur son souci d'équité spatiale à travers l'implantation des anganwadis et des écoles primaires à proximité de tous, les ONG, les associations, les institutions privées comme les particuliers ont pu occuper une place prépondérante dans un secteur peu réglementé. Ces structures ne sont pas soumises aux obligations de programmes, d'encadrement, de minima sanitaires... ni aux contrôles de la gestion de leurs fonds. Les familles s'enthousiasment du contraste qu'elles offrent avec les structures publiques et beaucoup soulignent leur efficacité. Les structures privées ont su bénéficier des effets positifs des programmes de scolarisation antérieurs ou de la création d'infrastructures en se localisant plutôt en zone urbaine ou le long des axes de communication (Ponceaud-Goreau, 2013). Elles ont une réelle réactivité face au processus de métropolisation et s'implantent plus rapidement dans les zones résidentielles attractives et les petites villes incorporées par les métropoles. La visibilité de cette offre, parallèle au système public, pourrait laisser à penser que l'État s'est lui-même retiré.

Pour illustrer la manière dont tout cela agit sur les processus de décision publique et comment l'État cherche à se repositionner, l'analyse se base plus particulièrement sur deux exemples : les villes de Chennai (État du Tamil Nadu) et de Pondichéry. L'État du Tamil Nadu et le Territoire de Pondichéry apparaissent précurseurs par la mise en place de politiques éducatives efficaces favorisant l'accès à la scolarisation, notamment des populations les plus modestes et des filles. L'État représente ainsi les intérêts des classes "subalternes", mobilisées depuis les années 1950 et valant par leur poids électoral. Des mesures sont devenues emblématiques comme la construction d'écoles dans chaque commune de plus de 1000 habitants, la distribution de repas chauds et gratuits, d'uniformes, de cartables et de manuels scolaires à tous les écoliers (Duraisamy et al., 1997). Parallèlement, la politique économique du gouvernement du Tamil Nadu a largement encouragé la croissance économique de la métropole de Chennai en réorganisant d'anciennes activités (chimie, sidérurgie, textile et cuir) et en en développant de nouvelles : technologies de l'information et industries automobiles (Marius-Gnanou, 2010). Le niveau d'éducation élevé et une offre d'emploi qualifiée ont également favorisé le processus de métropolisation favorable à la préscolarisation des classes moyennes. Les familles souhaitent investir dans l'éducation de leurs enfants et bénéficient d'une offre importante qui s'adapte, on le verra, à leurs contraintes sociospatiales. Les structures s'implantent particulièrement dans les petites villes qui entourent la ville-centre, aujourd'hui incorporées à cette dernière et enregistrant les plus forts taux de croissance (Durand-Dastès, 2012). Les intérêts des familles convergent aujourd'hui vers les différentes structures privées : attractivité de celles-ci, caractère répulsif du secteur public pourtant bien implanté, changements d'école durant le parcours scolaire et espoir d'une mobilité sociale. Les mobilités offertes sont nombreuses, créant une impression de vertige dans l'esprit des familles qui élaborent leurs stratégies. 
4 L'exemple de Pondichéry illustre un autre type de situation. L'offre scolaire est bien implantée et la ville constitue un pôle éducatif attractif, mais de nouvelles structures privées apparaissent et modifient le paysage éducatif. L'État cherche à se repositionner dans le secteur préscolaire grâce à un nouveau management offrant un accès au système privé pour les familles les plus modestes. Cela prend la forme de subventions, de paiement de salaires d'enseignants, de dotations de matériel dans un grand nombre d'écoles privées des centres-villes. A cela s'ajoute un certain nombre de changements comme l'ouverture des écoles primaires publiques aux préscolaires, le recrutement sous contrat précaire d'enseignantes formées aux jeunes enfants, et un enseignement moins magistral et plus participatif grâce à la chute spectaculaire des effectifs. Cet art du grand écart exige une belle souplesse de la part de l'État pour réellement espérer que tous les jeunes enfants puissent bénéficier des avantages d'une préscolarisation réussie. Initiateur du secteur préscolaire en Inde du Sud, l'État peut-il reconquérir les familles? Peut-il encore influer sur les logiques d'implantation des écoles privées? Quel rôle les classes moyennes supérieures peuvent-elles jouer dans l'orientation sociospatiale des preschools?

5 La démonstration s'appuie sur un travail d'analyse micro-géographique mené lors d'études de terrain dans des quartiers de Chennai et de Pondichéry en 2013 et 2014 et sur la collecte de témoignages. Ces entretiens, conduits auprès d'une trentaine de parents, d'employées de quatre anganwadis, d'enseignantes et de directeurs de dix-huit écoles publiques et privées, éclairent ce phénomène récent de grande ampleur. Ils sont retranscris en italiques, les parties entre parenthèses provenant d'autres moments de l'entretien.

\section{Evolution du système public: encourager l'initiative et relever les signes d'une crise de confiance}

6 Le Tamil Nadu a été particulièrement réactif aux directives de New Delhi, rendant accessible les services des anganwadis au plus grand nombre, avec 54439 centres en 2010. Les enfants bénéficient d'un mode de garde gratuit et sécurisé auquel s'ajoute la distribution de nourriture, le suivi de leur santé et une préparation à leur futur métier d'élève. En séduisant les électeurs par des investissements publics visibles, ces programmes catalysent également les revendications. La répartition des centres a été progressive mais le système est réellement précurseur, et répond aux exigences actuellement encouragées par l'UNESCO et les grands bailleurs de fonds internationaux, à savoir une approche qui combine nutrition, santé, soin et éducation auprès d'un personnel qualifié, et envers une population ciblée pour optimiser les dépenses publiques. L'État recrute et forme ses travailleuses sociales, oriente les services et l'enseignement dispensé, gère l'aspect matériel avec l'aide des collectivités locales et ciblent les populations les plus défavorisées (Gupta, 2012, p. 244). 
Illustration 1 - Shanti et son auxiliaire accueillent dix-huit enfants de 2 à 4 ans, dont une dizaine quotidiennement, Ganesh Nagar, Pondichéry

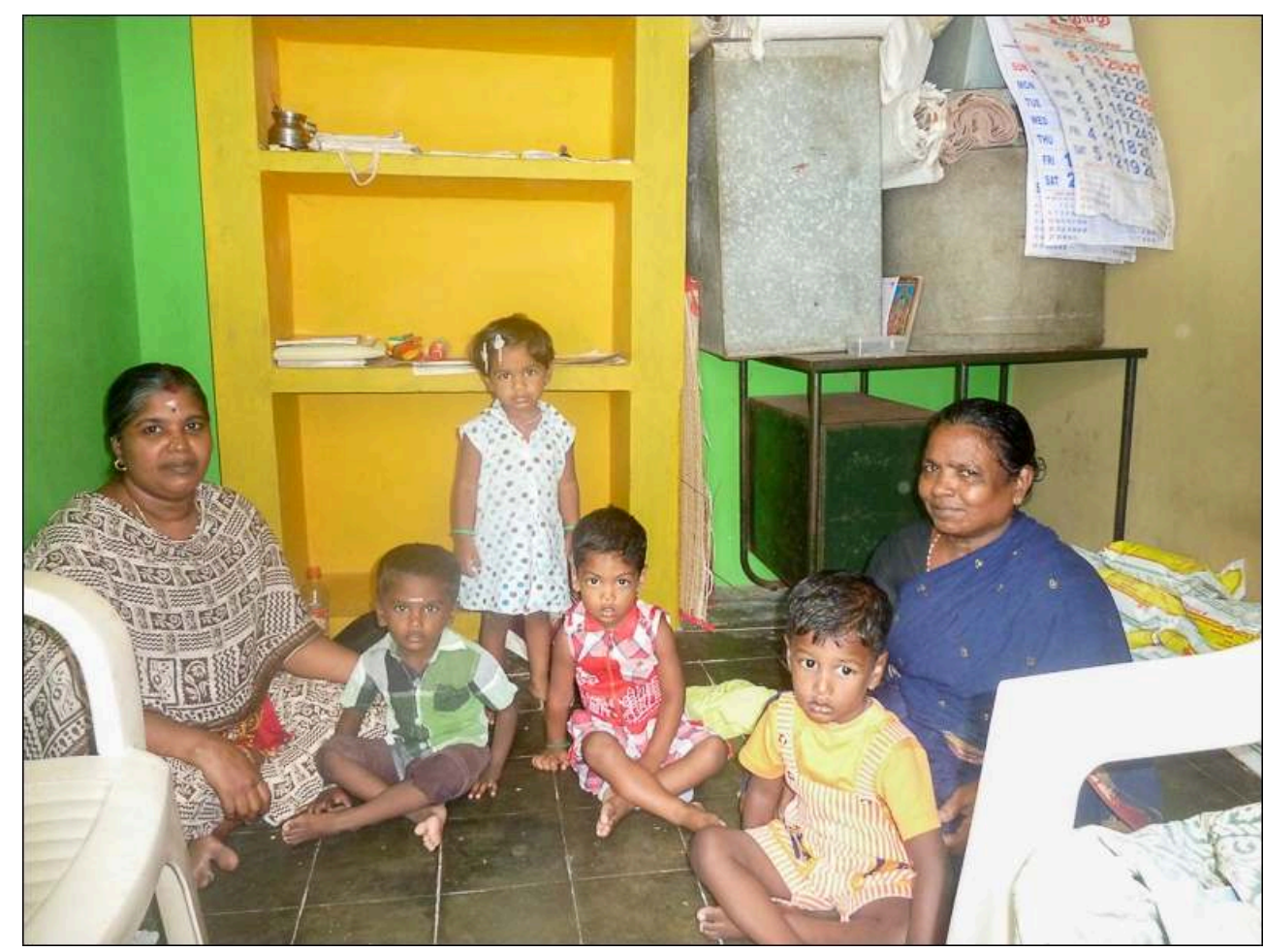

Ce jour-là, ils n'étaient que quatre car les autres étaient souffrants.

Auteur : E. Ponceaud-Goreau, 17 juillet 2014.

Entretien 1 avec Shanti, employée Anganwadi, à Muthialpet, Pondichéry, le 24 juillet 2014 :

Je travaille comme enseignante depuis 1985. À l'époque très peu de femmes voulaient travailler à l'extérieur de chez elles. Les anganwadis étaient plutôt situés en zone rurale, ils employaient des femmes du village mais en ville c'était plus difficile de recruter des volontaires. Moi, je venais de finir mes études secondaires, j'ai eu une formation de six mois spécifique à la petite enfance. Mon salaire mensuel n'était que de $250 \mathrm{Rs}^{3}$, et l'auxiliaire, 90 Rs. Je n'habitais pas très loin, encore jeune fille, mon père était d'accord pour que je travaille après mes études, surtout que c'était un emploi public. Les mères travaillent comme vendeuses de poissons, elles reviennent les chercher vers midi (...). Le loyer pour ce local est de 1000 Rs ; il n'y a qu'une pièce avec l'électricité mais sans eau courante ni toilettes. Le gouvernement ne nous donne que 500 Rs et nous (l'enseignante et son assistante) donnons le complément. Pour payer l'électricité on demande un peu aux parents. Cela ne nous dérange pas car nous travaillons ici depuis longtemps, mon salaire est de 23000 Rs. Les nouvelles enseignantes ont des contrats plus précaires et gagnent à peine quelques milliers de roupies.

7 Les employées de l'anganwadi devaient être des femmes recrutées localement pour s'occuper des enfants dont elles étaient proches. Le salaire garanti, le statut de fonctionnaire et la possibilité de travailler près de chez soi ont pu rendre ces emplois attractifs, et nombre d'entre eux ont été cooptés par les notables locaux pour leurs femmes ou leurs filles. Aujourd'hui cependant, le nombre croissant de jeunes filles diplômées et à la recherche d'un emploi auprès des jeunes enfants, a fortiori s'il s'agit d'un contrat public, permet à l'État de changer son mode de recrutement. Les contrats deviennent plus précaires, les salaires moindres et la mobilité des employées entre leur 
domicile et leur lieu d'exercice plus fréquente, ce qui influe parfois négativement sur l'ouverture quotidienne du centre.

8 De leur côté, les parents sont plutôt méfiants envers ces structures qu'ils considèrent comme de simples garderies, dissuadés par la mauvaise publicité véhiculée par les medias. Les centres anganwadis souffrent des dysfonctionnements reprochés aux écoles publiques : retards ou absentéisme des enseignantes, horaires aléatoires, locaux peu adaptés, absence de matériel... Des parents se mobilisent pour l'ouverture d'un centre quand d'autres centres sont moribonds dans des quartiers voisins. Le seuil est approximativement fixé à une cinquantaine de nouveaux foyers pour une ouverture, et le Territoire de Pondichéry a dû récemment créer une centaine de centres pour remédier aux manques dans les zones à forte croissance comme Muthialpet. Ce système qui paraissait souple dans ses fonctions et par les promesses d'une répartition équilibrée au niveau du territoire et d'un recrutement local, est aujourd'hui en pleine réorganisation. Les enfants de trois ans sont théoriquement encore accueillis toute la journée dans les centres anganwadis, mais les repas cuisinés sur place ont été remplacés à Pondichéry par la distribution bimensuelle de farine protéinée que les parents préparent à domicile. Le repas du midi n'étant plus fourni, les parents sont obligés de venir chercher leurs enfants vers midi et de les ramener éventuellement jusqu'à la fermeture du centre vers 15 heures. Cela est particulièrement contraignant pour les mères qui éduquent seules leurs enfants: souvent employées comme servante ou cuisinière, elles sont obligées de s'absenter pour aller les chercher et préfèrent alors souvent les emmener avec elles sur leur lieu de travail. Cela ne peut donc être un mode de garde efficace pour de nombreux parents, et le taux de présence effective est bas par rapport aux inscriptions. Depuis 2006, les gouvernements du Tamil Nadu et de Pondichéry ont décidé que les enfants de quatre et cinq ans seraient désormais scolarisés à l'école primaire voisine, qui doit être située à moins d'1 km d'un foyer d'habitations.

Entretien 2 avec le directeur de la Government primary school (GPS), à Pondichéry, le 7 août 2014 :

L'école [primaire] a été créée en 1975, il y a cinq professeurs et un directeur, quarante-six enfants pour sept niveaux. L'école est ouverte dès le Low Kinder Garden, mais il n'y a aucun enfant de cet âge, ensuite il y a six élèves en Upper Kinder Garden, une enseignante et une personne qui aide la maitresse. Cela fait peu, mais de manière générale les effectifs sont très bas, les parents se focalisent sur les écoles privées, surtout que beaucoup d'entre elles reçoivent de l'argent de l'État. Dans les faits, les meilleurs professeurs ont été élèves des écoles du gouvernement, mais les écoles privées font beaucoup de publicité à la télévision et dans les journaux. Les parents veulent que leurs voisins sachent qu'ils peuvent leur offrir une bonne éducation : l'uniforme (différent de celui de l'école publique), la carte d'identité de l'établissement en bandoulière, les belles chaussures, une cravate... La première chose à faire pour l'État est d'inspecter les écoles ouvertes illégalement et de réguler le système. En comparaison, les écoles publiques rurales sont mieux dotées, il n'y a pas de concurrence. 
Illustration 2 - Section preschool de l'école publique située à une quinzaine de kilomètres à l'ouest de Pondichéry

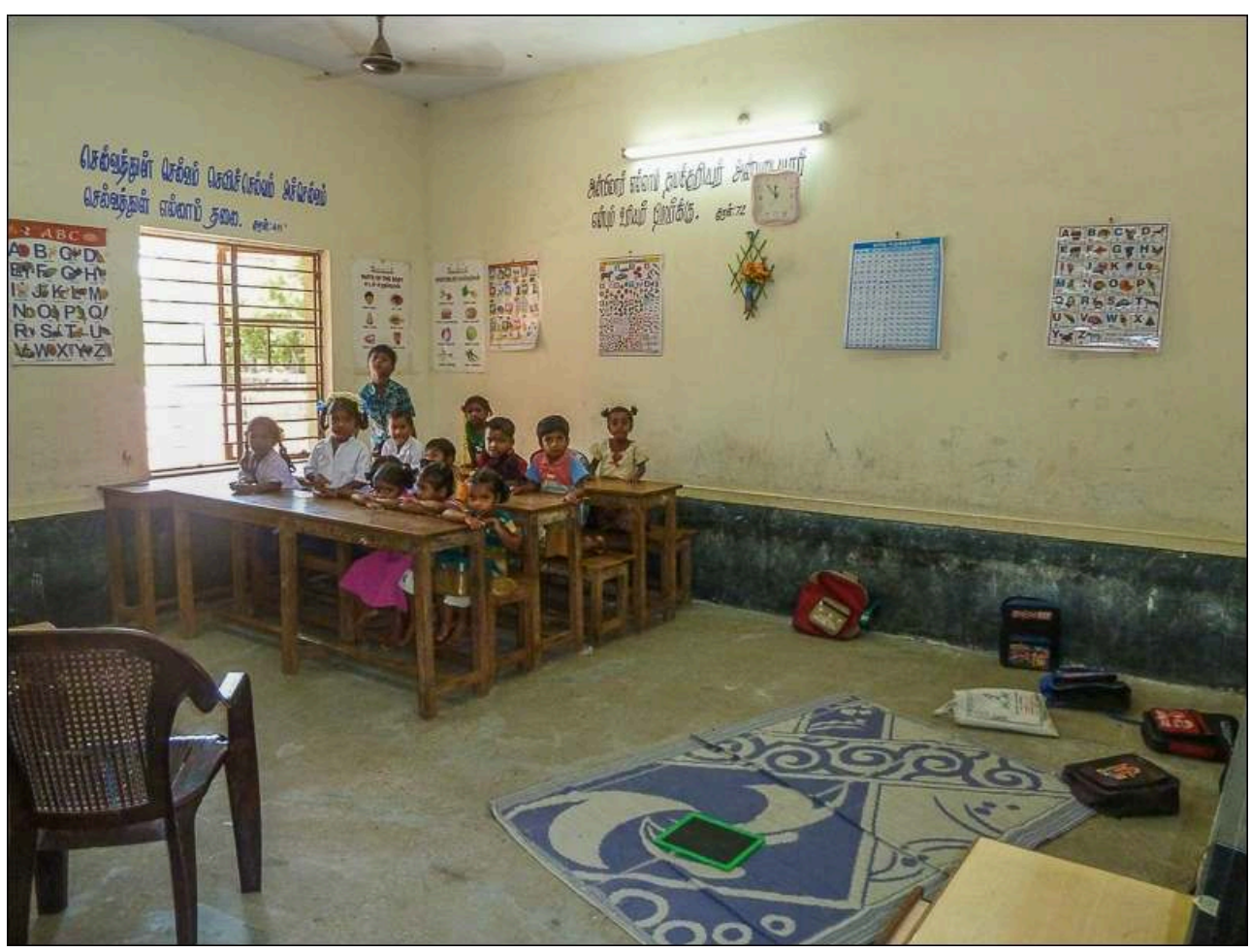

Les bureaux sont ceux utilisés par les élèves de la première année de primaire, les enfants du préscolaire s'installant habituellement sur les nattes.

Auteur : E. Ponceaud-Goreau, 17 juillet 2014.

Les écoles primaires sont contraintes d'accueillir les jeunes enfants. Régulièrement réparties sur le territoire, elles devaient offrir une solution adaptée aux contraintes des parents. Le personnel recruté pour la preschool n'est pas considéré comme personnel enseignant et ne bénéficie pas des avantages de celui-ci, notamment en ce qui concerne le salaire. La précarisation du contrat et la mutation vers un lieu d'exercice parfois assez éloigné rend ce personnel lui aussi assez désabusé. Le programme s'inspire assez librement $\mathrm{du}$ "système Montessori ${ }^{4}$ ", prônant un développement holistique de l'enfant qui respecte les rythmes d'apprentissage tout en favorisant la socialisation. Cependant, l'absence de matériel adapté aux tout-petits est mise en avant par les enseignantes tout comme le manque de locaux, et parfois d'accès aux sanitaires (entretien 2 et illustration 2). La promiscuité est grande : les élèves de première et deuxième années partagent le plus souvent la même salle, séparés seulement par une cloison mobile. Cet accueil pourtant gratuit et de proximité ne répond pas aux attentes actuelles des parents qui élaborent des stratégies de tout ordre pour éviter l'offre de préscolarisation publique, usant de leur capital financier autant que social. La proximité du domicile devient un critère négligeable dans leurs choix éducatifs.

\section{Le secteur privé contribue-t-il à une meilleure répartition de l'offre préscolaire?}

10 L'État s'est heurté à un certain nombre de contraintes face à son désir d'encourager la préscolarisation dans les écoles publiques. La lenteur administrative comme le prix du 
foncier en ville a limité l'expansion des écoles, ainsi que l'ouverture de nouvelles dans les zones résidentielles les plus attractives. La mise en place d'un partenariat publicprivé à travers le statut d'école privée sous contrat a permis à de nombreuses familles modestes d'accéder aux écoles privées et de soulager rapidement l'État. Ces établissements suivent le règlement des écoles publiques que ce soit pour le programme, le matériel ou les examens, et sont normalement ouverts sans critères d'entrée. Ce partenariat se différencie de l'affirmative action qui encourage la mixité sociale par la réservation de places dans l'enseignement secondaire et supérieur pour les enfants issus de basses castes, des castes et tribus répertoriées (Frisancho Robles, Krishna, 2012, p. 9). Il s'apparente davantage à un nouveau management public, qui dans ses efforts pour réformer l'administration, utilise les idées importées du secteur privé. En s'adossant au privé au Tamil Nadu, le secteur public continue à se repositionner, comme par exemple avec la préscolarisation et plus récemment l'introduction de l'anglais dès l'école primaire publique au Tamil Nadu.

Entretien 3 avec Kala, mère de famille à Pondichéry, le 22 juillet 2014 :

J'ai trois sœurs et ma mère travaillait comme servante, elle vit toujours ici (la maison d'une trentaine de mètres carrés est séparée par une demi-cloison, l'eau est disponible au robinet public). J'ai étudié jusqu'à 16 ans mais je n'ai pas eu l'examen final, je suis partie travailler comme servante. Mon mari, qui est vendeur de fruits, n'est pas allé à l'école, je m'occupe de tout. C'était un mariage arrangé et au départ j'ai vécu quelque temps avec sa famille du côté de Villuparam, mais je n'arrivais pas à vivre là-bas, il a vendu la terre et nous sommes revenus à Pondichéry. C'est mieux aussi pour les enfants de vivre en ville. Quand je me suis mariée il y a onze ans, je ne savais pas comment bien m'occuper des enfants. Quand ils ont été scolarisés, cela m'a permis d'échanger avec d'autres mamans. J'ai été scolarisée dans une « école du gouvernement » et je ne veux absolument pas que cela soit le cas pour mes enfants, surtout pas la «Tamil medium school». Je suis contente que Jayarani soit une école subventionnée, je ne paye les frais qu'une fois dans l'année (cette année, 3000 Rs pour sa fille en $1^{\text {ère }}$ année, 3100 Rs pour son fils en 5th), cela me permet d'économiser en prévision des études à venir et de leur payer des cours du soir (de 100 à 150 Rs par mois avec une étudiante de l'Université de Pondichéry qui vit près de chez eux).

$\mathrm{Au}$ début, je trouvais que l'école ressemblait à une prison mais les autres mamans m'ont expliqué qu'il était temps que mon enfant aille à l'école, que les enseignants savaient ce qu'il faut faire pour capter leur attention. Le fait que ce soit chrétien ne change rien pour moi, l'enseignement et les professeurs sont bons, c'est très peu cher et tout près de chez nous (il y a une école publique encore plus près). Parfois je me dis qu'il faudra peut-être changer d'école car dans le quartier beaucoup de leurs copains en partent mais c'est tout de même assez compliqué. Il faut aller voir le tehsildar officer (le préfet de canton) et le revenue inspector qui donnent les certificats de caste et de revenu, et le certificat de naissance. Je ne suis pas sûre de pouvoir remplir tous ces papiers, de pouvoir fournir les justificatifs et donner le dossier complet au département de l'Éducation 5 .

11 S'il y a toujours eu beaucoup d'acteurs présents dans le champ de l'éducation en Inde, les institutions catholiques ${ }^{6}$ sont particulièrement présentes dans le centre de la ville de Pondichéry. Leur enseignement est très recherché et leur offre scolaire suffisamment diversifiée pour correspondre aux différentes possibilités d'investissement des familles. Déjà présentes auprès des plus défavorisés, elles sont préoccupées par l'idée d'inculquer aux enfants des familles les plus modestes un certain nombre de "bonnes pratiques ", et mettent en avant dans leurs discours la nécessité d'éduquer ces enfants dont les parents sont « non éduqués » (Delpeu, 2011). Plus encore que dans les écoles publiques, elles encouragent les enfants à adopter une bonne hygiène par le lavage régulier des mains et des visages et à prendre soin de leur 
uniforme, elles corrigent les postures du corps, maintiennent une certaine discipline et n'hésitent pas à conseiller les parents. Elles savent aussi s'arranger avec les associations qui dirigent de plus petites structures en prenant leurs protégés si, par accord tacite entre les directeurs, leurs frais sont financés par un parrainage. Les familles, comme le montre l'entretien avec Kala, sont particulièrement enthousiastes à l'idée que leur enfant soit scolarisé dans une école privée, qui plus est une institution catholique, réputée pour la qualité et le sérieux de ses enseignantes et leurs formations. Les parents n'hésitent pas à parcourir des distances parfois importantes si leurs enfants ont été acceptés dans une de ces institutions du centre-ville. L'État, comme les associations, en jouent pour motiver l'intérêt des familles envers l'Éducation et les y engager le plus tôt possible. Comme les structures existaient déjà, l'État leur a sous-traité une grande partie de la préscolarisation des plus pauvres. Il n'a donc pas eu beaucoup d'influence sur leur localisation, et il est à noter que l'absence de contraintes formelles pour l'accueil des jeunes enfants a permis à de nombreuses écoles privées du centre-ville de Pondichéry d'accueillir plus d'élèves sans pour autant améliorer leurs équipements sanitaires ni fournir de repas, de matériel ou de jeux adaptés. Seules 15 preschools fonctionnent indépendamment d'une école primaire tandis que 186 écoles privées ont intégré une section préprimaire à leur école déjà existante. Par la suite, les contraintes administratives rebutent souvent les parents qui pourraient envisager de changer d'établissement.

Les écoles dirigées par des associations ou des ONG sont également très nombreuses. Ces écoles se situent plutôt dans des zones nouvellement construites, aux terrains peu chers, ciblant une population telle que les pêcheurs qui ont perdu leurs maisons à cause du tsunami comme à Muthialpet, les familles monoparentales, ou des populations nouvellement implantées. Pour d'autres établissements, le lieu est lié à l'histoire personnelle de son fondateur, parfois politicien local souhaitant œuvrer pour son électorat, ou action philanthropique d'un individu ayant connu un succès financier. Ces écoles combinent pragmatisme et autorité morale orientée vers la réalisation de l'objectif du Millénaire sur l'éducation et la protection de la petite enfance (Lewis, Mosse, 2006, p. 8). Certaines disent avoir profité de "l'effet tsunami » après 2004 : beaucoup d'argent a été mis à leur disposition suite aux campagnes de dons, et la construction d'une "école pour les jeunes enfants" était une image parlante pour sensibiliser les donateurs à leurs actions plus générales sur le terrain. Elles «communiquent" sur la participation de volontaires étrangers venus dans leur preschool. L'État ne peut réguler cette offre; les institutions ne sont pas vraiment contrôlées, dans la mesure où elles ne s'agrandissent pas en ouvrant des sections de l'école primaire et dans ce cas, le contrôle se résume souvent à une simple visite au moment où elles demandent le certificat qui leur permet de recevoir des fonds étrangers, lequel est facile à obtenir. Ces écoles sont des structures parallèles à celles de l'EÉtat et bénéficient d'une grande liberté d'installation. Le Territoire de Pondichéry est particulièrement attractif dans l'imaginaire étranger.

Structures privées et structures publiques sont visibles sur l'ensemble du Territoire ${ }^{7}$ mais elles offrent un contraste saisissant aux yeux des familles. Les écoles privées bénéficient de l'image dévalorisée des écoles primaires publiques où sont déplorés entre autres les forts taux d'absentéisme des enseignants, le manque de temps effectif d'apprentissage ou la dévalorisation des élèves (Rapport $\mathrm{PROBE}^{8}$, 1999). Les établissements subventionnés ou gérés par des associations proposent une certaine 
qualité qui focalise l'attention des familles comme l'enseignement de l'anglais en $L K G$ et UKG, extrêmement valorisé dans la suite du cursus ${ }^{9}$; il existe aussi une certaine attraction pour les écoles gérées par des occidentaux ou des religieux. La concurrence est présente à tous les niveaux mais les familles les plus modestes constituent la cible la plus importante et de nombreuses écoles profitent de leur engouement pour la préscolarisation. Le déplacement quotidien des jeunes enfants est aujourd'hui rendu possible par l'usage de plus en plus fréquent de la moto qui évite les trop longues marches. Les pères n'hésitent plus à demander des temps de pause qui correspondent aux horaires de l'école pour assurer le transport des petits, les leurs mais aussi ceux de leurs voisins ou de leur parenté, et les ramener à la maison auprès de leur mère avant de repartir travailler. Alors que l'école publique favorise les relations de proximité, se tourner vers les structures privées même plus éloignées est aujourd'hui possible car les parents ont modifié leur représentation des distances. Les changements de représentations spatiales des lieux fréquentés par l'enfant viennent accélérer le processus de métropolisation. L'école se rapproche également des parents comme les structures à bas coût ${ }^{10}$ qui se développent dans les petites et moyennes villes, tandis que d'autres choisissent de s'implanter le long des axes routiers, à la campagne ou dans les bourgs

\section{Illustration 3 - École à Kudapakkam}

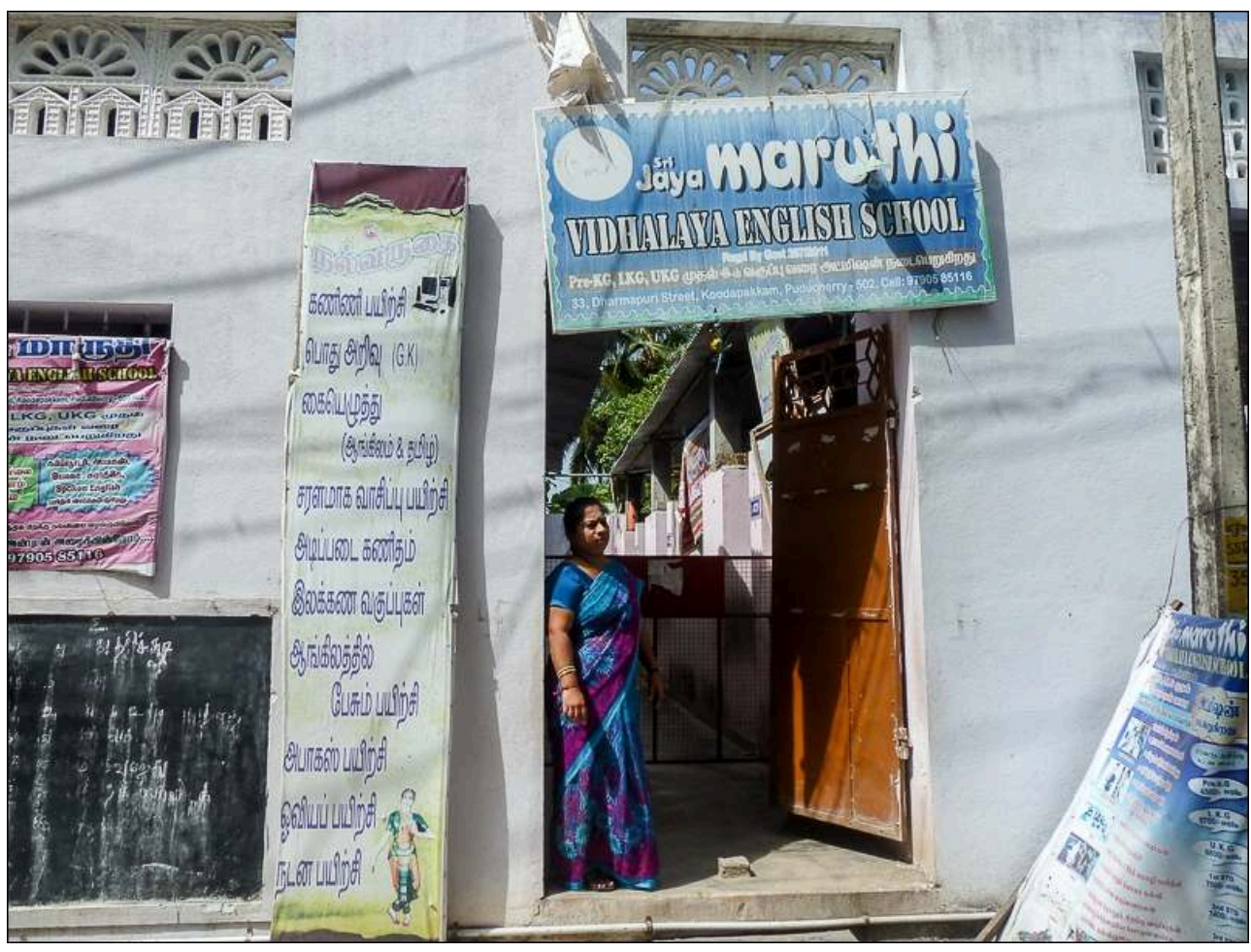

Auteur : E. Ponceaud-Goreaud, 17 juillet 2014.

Entretien 4 avec la maîtresse et directrice d'une école à Kudapakkam, le 17 juillet 2014 :

Il y a 25 élèves et nous pouvons enseigner du $L K G$ au niveau $6^{\mathrm{e}}$ année avec l'autorisation du gouvernement. En ce moment, c'est compliqué parce que l'autre maîtresse s'est mariée et ne vient plus. Je suis toute seule pour tous les niveaux. Mon mari s'occupe plutôt de la partie marketing, il a créé la plaquette publicitaire et va bientôt recruter une nouvelle enseignante. Nous habitons à $2 \mathrm{~km}$ à Villianur. 
Nous nous sommes installés ici car mon mari est natif du village. C'est pourtant assez concurrentiel. Juste à côté, il y a une grosse école (à $100 \mathrm{~m}$ ) qui existe depuis 35 ans avec près de 1000 élèves, un peu plus loin à gauche une autre pour les riches, un peu plus loin une autre vient de fermer faute d'élèves. Mais moi, j'ai toujours été très motivée par l'idée d'ouvrir une école depuis que j'ai obtenu mon diplôme universitaire en éducation.

Un particulier disposant d'un peu d'argent à investir, d'un terrain ou d'une maison vacante peut ouvrir une preschool (entretien précédent et illustration 3). L'obtention d'un diplôme lié à l'éducation est un " plus » mais son absence n'est pas rédhibitoire. Les propriétaires de petites structures profitent d'une main d'œuvre féminine nombreuse et qualifiée grâce aux progrès de l'enseignement secondaire public, et qui accepte des salaires faibles. Ces jeunes filles occupent ces emplois entre la fin de leurs études et leur mariage, accédant à une certaine mobilité sociale tout en exerçant un métier " convenable » (Ponceaud-Goreau, 2014). Ces structures font partie aujourd'hui du paysage éducatif grâce aux élites qui ont orienté son développement en sollicitant les acteurs privés, suivis par un nombre très important de familles.

\title{
Quel rôle les classes moyennes supérieures peuvent- elles jouer dans l'orientation socio-spatiale des structures préscolaires?
}

\begin{abstract}
Entretien 5 avec Kesang Menezes ${ }^{11}$, à Thyruvanmiyur, Chennai, le 10 juillet 2013 : Les parents sont très inquiets de connaitre les conditions favorables à la réussite scolaire, ils discutent beaucoup de ce sujet et élaborent des stratégies en fonction des différents conseils et récits d'expériences qu'ils peuvent collecter. Les parents pensent que c'est bon pour les enfants d'aller tôt à l'école : plus ils étudient tôt, plus ils pourront apprendre. Les classes moyennes poussent beaucoup leurs enfants. Et puis, si tu regardes il y a 50 ans, il y avait beaucoup d'enfants, les familles étaient grandes, les enfants dépensaient leur énergie. Maintenant, les parents disent qu'ils ne savent pas quoi faire avec eux à la maison (...). La mobilité au quotidien n'est pas vue comme une contrainte s'il s'agit pour leur enfant d'accéder à la meilleure école possible. Des fois, on les envoie à $10 \mathrm{~km}$ de chez eux parce que c'est une école réputée. D'ailleurs, les parents cherchent avant tout à être dans la preschool associée à la bonne école primaire, ils sont sûrs que leur enfant pourra y poursuivre sa scolarité. Après, comme tu as payé les frais, tu y laisses ton enfant, tu ne leur demandes pas ce qui s'y passe.
\end{abstract}

Les classes moyennes souhaitent consolider voire améliorer leur position à travers un processus de mobilité sociale et leurs choix éducatifs se placent au premier plan de leurs préoccupations. Les entreprises de services comme les preschools privées s'installent dans les quartiers résidentiels pour répondre aux besoins des familles. Le mode de vie urbain a, dans un premier temps, correspondu à l'élaboration d'un processus identitaire d'appartenance à la classe moyenne et d'un désir d'en afficher ses marqueurs (Fernandes, 2006, p. 80). La fréquentation d'une bonne preschool en est un. Les familles les plus riches n'ont jamais voulu fréquenter les services de préscolarisation publique. Leur absence du système éducatif public a fait que les personnes les plus à même de protester, demander des comptes, s'organiser et trouver des appuis politiques pour soutenir leurs demandes n'ont jamais eu l'occasion de faire pression sur les pouvoirs publics pour obtenir l'amélioration de l'enseignement, que ce soit en ville ou à la campagne (Majumbar \& Mooij, 2011, chap.2). Elles se sont directement orientées vers l'offre privée qui a profité de l'absence de régulation du 
système pour imposer un certain nombre de règles dans les preschools. Les frais engagés ${ }^{12}$ rendent les parents plus exigeants et plus actifs. De la même manière, les frais d'écolage agissent comme une barrière d'entrée pour certaines familles, et ils sont une barrière de sortie pour les autres qui préfèreront interférer dans la politique de l'école plutôt que de risquer de perdre leur «investissement " (Majumdar \& Mooij, 2011, p. 75).

Entretien 6 avec Mansi, mère de deux enfants de 3 ans et 5 ans, à Thyruvanmiyur, Chennai, le 31 juillet 2014 :

Les étapes pour trouver une bonne école? D'abord il faut récupérer un des formulaires qui sont édités de manière limitée, il faut souvent envoyer un chauffeur (d'auto-rickshaw ${ }^{13}$ ) exprès, qui fait la queue. Après on vous appelle pour un entretien. Cela peut être des tests informels, voir si l'enfant est à l'aise avec les étrangers ou carrément un entretien avec les parents et l'enfant. Lors de l'entretien, on demande surtout aux parents où est-ce qu'ils travaillent, l'importance que revêt l'éducation pour eux et la part financière qu'ils comptent y investir. Le gouvernement a essayé de réguler les frais d'écolage mais les mesures sont passées inaperçues ${ }^{14}$ (...) J'ai arrêté de travailler (dans une banque) car nos parents habitaient trop loin pour s'occuper de mes enfants. Les domestiques classiques ne peuvent éduquer correctement un enfant, leur langage, leur vocabulaire ne seront pas bons. Elles s'en occuperont mal, ne sauront pas ce qu'il faut lui donner de bon à manger ou le faire jouer correctement. Elles les aiment bien sûr et veulent leur faire plaisir alors elles les laissent regarder la télévision en permanence et leur donnent trop de sucreries. Moi, je sais ce qu'il faut faire avec mon enfant, comment lui parler d'un livre par exemple.

L'arrêt momentané ou prolongé de l'activité professionnelle de la mère correspond à la majorité des situations vécues par les familles urbaines. Le désir de s'occuper de son enfant et d'habiter le rôle traditionnel de mère, mais aussi l'absence de "famille élargie » et la difficile conciliation du travail féminin et de la journée d'école sont les causes les plus fréquentes. L'accueil périscolaire, qui serait une solution à la continuité de l'emploi de la mère, est encore assez rare et ne présente qu'une alternative temporaire car peu de familles envisagent d'y laisser leur enfant si longtemps. Les structures qui se développent dans les zones urbaines aisées correspondent plutôt à des preschools ouvertes entre $9 \mathrm{~h}$ à $12 \mathrm{~h}$ : les familles se disent soucieuses de l'épanouissement de leurs enfants mais sans contrarier leur rythme naturel. Quelques services annexes fleurissent dans ces quartiers comme les cafés où les mamans et leurs enfants se rencontrent, les salles de jeux climatisées ou, récemment, les agences d'employées de maison spécialisées dans la garde d'enfant à domicile. Celles-ci ont été «formées » à parler aux enfants, jouer avec eux, les nourrir correctement avec des aliments sains ou choisir les bons fruits pour leur collation : elles peuvent prétendre à des rémunérations plus élevées, de 7 à 8000 Rs au lieu de 3 à 4000 Rs pour les domestiques non formées. En cela, la réactivité des petites structures est remarquable ; cette forme est particulièrement présente dans les zones d'habitat résidentiel attractif où se concentrent les jeunes ménages de la classe moyenne supérieure. Ces preschools sont franchisées, aisément repérables dans le paysage et assurent une certaine idée de qualité. 


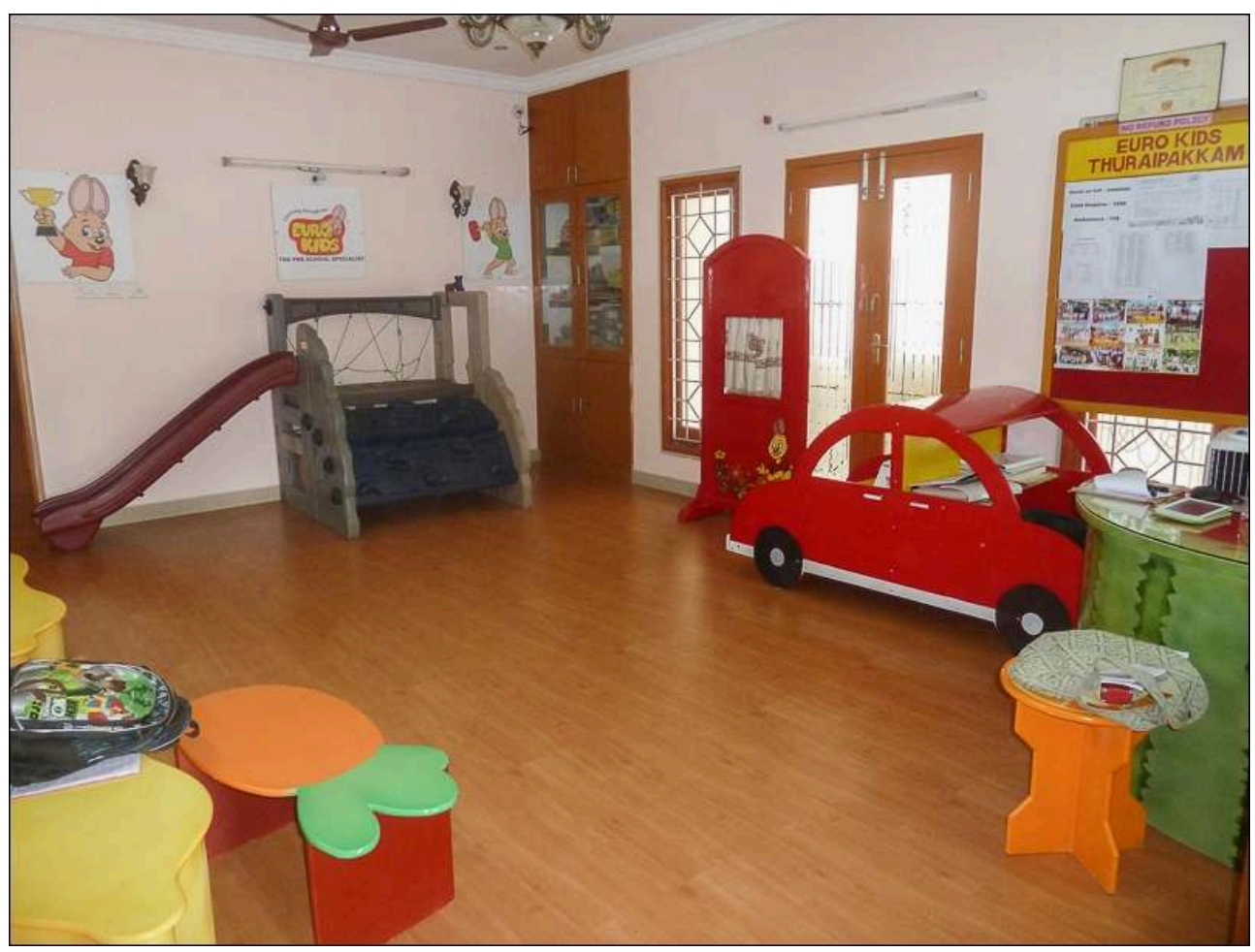

Créé en 2001, Eurokids est présent dans 311 villes d'Inde avec 884 établissements franchisés. Pour espérer être franchisé, il faut disposer dans un lieu agréable d'un local confortable d'environ $180 \mathrm{~m}^{2}$ et investir de 100000 à 150000 Rs. Le mobilier, la décoration, le matériel sont standardisés.

Auteur : E. Ponceaud-Goreau, le 4 août 2014

La présence des enseignes franchisées se traduit par l'implantation rapide de preschool dans une maison ou un appartement; le matériel, le mobilier, la formation des enseignantes, la publicité étant assurée par le groupe (illustration 4). La concurrence est rude avec les écoles plus anciennes qui associent le primaire voire le secondaire, où les parents s'engagent dans un processus éducatif long et coûteux, obligés de se conformer aux exigences de l'école, de fournir des donations ... pour conserver l'avantage d'être dans l'établissement. Les nouvelles structures sont conscientes de l'importance de l'accessibilité et si elles ne peuvent s'installer au plus près des zones résidentielles aisées, elles visent l'installation près des axes routiers, renforçant le processus de métropolisation. Des structures plus grosses s'installent là où les terrains sont moins coûteux, construisant de véritables campus accessibles grâce à leurs bus privés. Les villes moyennes ne sont pas en reste comme le long de la route de Tirukanur à Koodapakkam, à $8 \mathrm{~km}$ de Pondichéry. À Chennai, la métropolisation a pris la forme de "corridors de développement» dans un contexte de planification foncière. L'État a encouragé les investisseurs privés à construire des lotissements résidentiels le long de ces corridors où les services spécifiques à ces familles aisées se sont à leur tour installés, comme le long de l'old Mahabalipuram Road (Marius-Gnanou, 2010). L'offre préscolaire privée y est particulièrement développée.

Entretien 7 avec Vidya Shankar, fondatrice de Montessori Cascade Center, à Chennai, le 28 juillet 2014 :

J'étais magistrate pour le Conseil de protection des mineurs mais je voyais bien que les enfants n'avaient pas accès à une éducation de qualité. J'ai fondé l'école en janvier 2011, il n'y avait que deux enfants et je louais seulement la pièce du sous- 
sol. Actuellement, il y a dix-huit enfants, dix grands (de 6 à 10 ans) et 8 petits (moins de 6 ans) et nous louons les trois étages (d'une petite maison) pour 60000 Rs par mois. Je souhaiterais être plus indépendante, mettre moins d'argent personnellement. Les trois enseignants ${ }^{15}$ ont reçu la formation au Center for Montessori Training (CMTC), à Adyar. L'environnement doit être amical, il faut être proche des enfants, ne pas trop les pousser. Pour nous faire connaître, nous organisons des camps d'été et du tissage le dimanche. Les gens du quartier peuvent découvrir l'école. Ce sont plutôt des parents de la classe moyenne supérieure, c'est important qu'ils discutent de l'école entre eux. Beaucoup de mères sont au foyer ou travaillent à temps partiel, elles aiment rester un peu à l'école, voir leur enfant évoluer et discuter avec l'enseignant.

Les quartiers résidentiels «chics » voient se développer un nouveau style de preschool où le développement sensori-moteur de l'enfant est mis en avant dans un cadre jugé " épanouissant ", avec beaucoup de jeux à disposition, des activités artistiques variées, un taux d'encadrement important, un service de restauration bio... La méthode Montessori est particulièrement valorisée dans ces petites structures, grâce à la présence de nombreux centres de formation à Chennai qui s'adressent aux adultes de tout profil souhaitant se spécialiser et par la vente de matériel coûteux en ligne. Ces preschools haut-de-gamme rejoignent les nouvelles méthodes pratiquées dans les écoles publiques même si les moyens diffèrent.

\section{Conclusion}

L'investissement éducatif est devenu une priorité familiale et la préscolarisation, comme l'enseignement de l'anglais et le choix de l'école privée, un de ses signes distinctifs. Adoptés par les classes moyennes, une part croissante de familles plus modestes s'efforce de les acquérir. Dès 1975, l'accueil des jeunes enfants a été au cœur des politiques publiques en faveur de la réduction des inégalités par la création des centres anganwadis, qui couvraient une large part de la population sur l'ensemble du territoire. Les progrès ont été significatifs dans les domaines de la santé et de la lutte contre la malnutrition, et le système constitue actuellement, selon les bailleurs de fonds et les institutions internationales, un modèle pour les nombreux pays du Sud engagés dans l'éducation et la protection de la petite enfance. La demande des familles indiennes s'oriente aujourd'hui davantage vers la scolarisation. Parallèlement, le gouvernement a été « épaulé » de manière hétérogène par le domaine associatif et les ONG. Leur part croissante dans le secteur de la préscolarisation a pu laisser penser que l'État s'était lui-même désengagé des familles les plus modestes, axant ses priorités sur le système éducatif primaire et secondaire. Pourtant, depuis 2006, l'État cherche à se repositionner en ouvrant l'école primaire aux préscolaires suite à la chute spectaculaire des effectifs dans de nombreuses écoles publiques et la mise en place d'une réflexion sur les méthodes d'enseignement. En réponse à la demande croissante des familles pour les établissements privés, l'État s'est positionné vers la mise en place d'un meilleur partenariat public-privé. Celui-ci reste lié au recrutement de jeunes femmes en contrat précaire et à la présence d'organisations déjà présentes auprès des plus modestes afin que la participation imposée aux parents ne représente pas plus de quelques centaines de roupies par mois.

Par l'importance de leur réseau scolaire, le Tamil Nadu et Pondichéry sont en mesure d'apporter une réponse efficace en matière d'offre préscolaire publique renforcée par leur maîtrise des relations de partenariat public-privé. Cette offre d'enseignement est 
assez rare en Inde et plus encore, dans un contexte mondial où les institutions et les bailleurs de fonds sollicitent essentiellement le secteur privé ; elle peut constituer un modèle alternatif intéressant où l'État redevient un régulateur efficace. Alors que la plupart des pays du Sud, qui visent la réalisation des objectifs du Millénaire, débutent la diffusion de la préscolarisation, l'expérience des anganwadis indiens est significative pour mesurer les efforts des politiques publiques envers les jeunes enfants. Le cas du Tamil Nadu et de Pondichéry renseignent plus particulièrement sur les réussites et les réajustements à prévoir lorsque les acteurs privés entrent en scène.

La diffusion de la préscolarisation n'est pas seulement un levier au développement qui permet de lutter contre les inégalités liées à la naissance dans un milieu défavorisé. Elle est aussi le reflet de l'importance de l'investissement éducatif des familles. Le changement de mode de vie a ouvert un champ des possibles dans les esprits des familles de la New Middle Class (Fernandes, 2006, p. 29) diffusant massivement la préscolarisation. L'implantation des structures privées s'est faite en scrutant les représentations des classes moyennes et celles, de plus en plus nombreuses, qui souhaitent en épouser le mode de vie : la distance domicile-école n'est pas rédhibitoire mais résulte d'un choix. L'engouement des familles modifie l'offre scolaire publique et complexifie la mise en place d'une certaine équité de l'offre éducative. L'étude du cas indien est en cela particulièrement instructif pour de nombreux pays qui encouragent la préscolarisation en ciblant leurs efforts sur les zones géographiques où les progrès seront les plus visibles. Les familles sont elles-mêmes porteuses d'un projet d'individuation et leurs stratégies peuvent modifier les effets attendus par les politiques. Les conditions et les enjeux du développement du secteur préscolaire, notamment dans les villes moyennes et petites, sont à mettre en relation avec les aspirations des familles et les stratégies qu'elles élaborent pour accéder à une certaine éducation de qualité. L'orientation donnée par les classes moyennes supérieures peut aussi orienter les réajustements à prévoir. Autant de pistes pour évaluer la politique de préscolarisation accomplie au Tamil Nadu et Pondichéry grâce aux actions conjointes d'un gouvernement porté par l'idée de progrès, de nombreux et réactifs acteurs privés, et des familles qui ont su exercer leur pression par le bas.

\section{BIBLIOGRAPHIE}

Buisson A., 2009. Alphabétisation et éducation en Inde, l'exemple du Kérala. Paris, L'Harmattan, 193 p.

Clémentin-Ojha C., 2008. Les chrétiens de l'Inde. Entre castes et Églises. Paris, Albin Michel, 300 p.

Delpeu M., 2011. Enfances, sida et religions en Inde du Sud : une ethnographie de la circulation des enfants séropositifs. Doctorat Ethnologie, Université Bordeaux Segalen.

Duraisamy P., James E., Lane J., Jee-Peng T., 1997. Is there a quantity-quality tradeoff as enrollments increase? Evidence from Tamil Nadu, India. Policy, Research working paper, $\mathrm{n}^{\circ}$ WPS 1768, Washington, DC, World Bank. 
Durand-Dastès F., 2012. La population indienne en 2011 : anciennes et nouvelles différenciations spatiales. Mappemonde, $\mathrm{n}^{\circ} 108$.

Gupta A., 2012. Red Tape: Bureaucracy, Structural Violence, and Poverty in India. Durham, Duke University Press, $384 \mathrm{p}$.

Fernandes L., 2006. India's New Middle Class: Democratic Politics in an Era of Economic Reform. Minneapolis, University of Minnesota Press, 336 p.

Frisancho Robles V., Krishna, K., 2012. Affirmative Action in Higher Education in India: Targeting, Catch Up, and Mismatch, NBER working paper.

Lewis D., Mosse D., 2006. Theoretical Approaches to Brokerage and Translation in Development. In Lewis, D., Mosse D., Development Brokers and Translators: The Ethnography of Aid and Agencies. Bloomfield, CT, Kumarian Press, p. 1-26.

Majumdar M., Mooij J., 2011. Education and inequality in India: a classroom view. New York, Routledge, $206 \mathrm{p}$.

Marius-Gnanou K., 2010. Nouvelles activités économiques et dynamique métropolitaine : le cas de la périphérie Sud de Chennai. Annales de Géographie, n 671-672, p. 28-51.

Ponceaud-Goreau E., 2014. Relations de genre dans l'enseignement préprimaire en Inde du Sud. Journée d'études Mixité et éducation : pratiques sociales et dimensions culturelles, Bordeaux, France, 16 mai 2014.

Ponceaud-Goreau E., 2013. La diffusion de l'enseignement pré-primaire au Tamil Nadu. Séminaire Jeunes Chercheurs de l'AJEI Passages, médiations, connexions en Asie du sud, Paris, France, 12 novembre 2013.

PROBE Team, 1999. Public Report on Basic Education in India. Oxford University Press: New Delhi.

Srivastava P., 2006. Private schooling and mental models about girls' schooling in India. Compare, vol. $36, \mathrm{n}^{\circ} 4$, p. 497-514.

UNESCO, 2007. Rapport Education et protection de la petite enfance. http://unesdoc.unesco.org/ images/0015/001500/150022f.pdf. Consulté le 25/09/2014.

UNESCO, 2013-2014. Rapport Enseigner et apprendre : atteindre la qualité pour tous. http:// www.unesco.org/new/fr/education/themes/leading-the-international-agenda/efareport/ reports/2013/. Consulté le 30/09/2014.

\section{NOTES}

1. Le terme preschool a été conservé ici ; il recouvre, dans un cadre peu règlementé, l'ensemble des systèmes publics et privés prenant en charge les enfants de 3 à 6 ans.

2. L'éducation est une compétence partagée entre le gouvernement central de Delhi, qui suggère les politiques éducatives et les programmes scolaires, et les États et Territoires qui ont leur propre ministère de l'Éducation, qui gèrent leur propre système scolaire avec leurs propres manuels et leur propre système d'évaluation. La loi du $1^{\mathrm{er}}$ avril 2010 vise à garantir un enseignement gratuit et obligatoire pour tous les enfants de 6 à 14 ans. Le parcours commence à l'école primaire qui est divisée en cinq classes appelées standards - un enfant scolarisé en $5^{\mathrm{e}}$ année d'école primaire est en 5 th. Dans les écoles primaires publiques, l'enseignement se fait dans la langue de l'État. Au Tamil Nadu, le cursus s'y fait donc dans des Tamil medium schools, souvent présentées en opposition avec les English medium schools privées. Depuis 2006, les 
élèves ont la possibilité d'effectuer une préscolarisation en LKG (low kinder garden) à partir de 4 ans et en UKG (upper kinder garden) à partir de 5 ans.

3. Au 12 octobre 2014 , le taux de change pour un euro est de 77,30 roupies, 250 Rs équivaut donc à 3,23 euros et 23000 Rs à 297,55 euros.

4. «L'enfant n'est pas un vase que l'on remplit, mais une source que l'on laisse jaillir ». Telle est la philosophie de Maria Montessori, médecin italien, dévouée à la cause des enfants, qui a séjourné en Inde pour fuir la seconde guerre mondiale. Ses idées, soutenues par le Mahatma Gandhi et Tagore, ont pu s'y développer et essaimer avec la création de milliers d'écoles dans tout le pays et plus spécialement une dizaine à Chennai. Le langage, l'écrit et les mathématiques sont abordés avec un matériel créé par Maria Montessori, mis à la disposition des enfants qui ont la liberté de choisir eux-mêmes les activités qu'ils souhaitent faire. Un éducateur adapte et stimule l'enfant en périphérie pour qu'il apprenne par lui-même, à son rythme.

5. Kala doit prouver que sa famille, appartenant aux castes non répertoriées, peut bénéficier des places qui leur sont réservées dans les écoles privées subventionnées. Une amie, qui connaissait la procédure, l'avait aidé à établir le dossier la première fois mais elle ne se sent pas capable de le faire seule.

6. Les chrétiens représentent $2,3 \%$ de la population indienne mais administrent près de la moitié des écoles subventionnées (Clémentin-Ojha, 2008, p. 29).

7. Les parts des preschools publiques et privées sont équivalentes à Pondichéry : 201 sont privées et 199 sont publiques; la proportion reste très proche si on ajoute Mahé, Yanaon, Karaikal qui font partie aussi du Territoire (278 écoles privées et 302 publiques).

8. Le projet PROBE est le fruit d'une collaboration entre l'Institute of Social Studies Trust, à New Delhi, et Collaborative Research and Dissemination (CORD) jouissant du financement du Centre de recherches pour le développement international (CRDI).

9. L'expression «English medium syndrome " traduit l'importance d'un phénomène commun à toute l'Inde: les parents disent retirer leur enfant des établissements publics principalement parce que l'anglais n'y est enseigné qu'à partir du collège.

10. Les écoles du secteur privé à bas coût (low fee private schools ou LFP) se multiplient de manière exponentielle depuis une dizaine d'années car elles sont très rentables pour leurs propriétaires. Les LFP peuvent se définir par leur auto-ciblage des groupes marginalisés ; elles sont entièrement financées par les frais d'écolage ; les frais pour un mois ne doivent pas dépasser le montant d'une journée de salaire pour un ouvrier agricole dans les primaire, jusqu'à deux journées pour l'enseignement secondaire (Srivastava, 2006).

11. Depuis 2004, elle organise des discussions entre de petits groupes de parents directement dans les entreprises. Elle écrit également des articles sur la parentalité notamment pour le Parent Circle Magazine et le quotidien The Hindu.

12. Les frais d'écolage d'une preschool varient, en fonction du public visé, de plusieurs centaines d'euros à une centaine de roupies par mois et sont payables mensuellement. Àcela s'ajoutent souvent des frais annuels d'inscription, l'achat de matériel, de cahiers, de manuels et d'uniformes, des abonnements à la bibliothèque, le transport en autocar ou en rickshaw...

13. Ce tricycle motorisé est très fréquent en Asie, sa partie avant est construite comme une motocyclette ou un scooter, tandis qu'à l'arrière se trouve une banquette pour transporter les passagers : cela représente une alternative intéressante aux réseaux de transport traditionnels, voire une solution à l'absence de ces infrastructures. Le chauffeur de rickshaw transporte des passagers comme un taxi ou par abonnement pour les transports d'enfants vers les écoles, il effectue également des livraisons et peut rendre de menus services.

14. La régulation des frais de scolarité au Tamil Nadu établit les frais d'écolage maxima autorisés pour les écoles privées pour 2013-2016 - consultable pour Chennai sur: http://www.tn.gov.in/ miscellaneous/pdf/31.pdf 
15. Deux femmes et un homme - le seul enseignant masculin de preschool rencontré sur les deux années de terrain. Issu d'une famille très pauvre, ses études ont été financées par une société privée. Il souhaitait enseigner comme professeur du secondaire dans une école publique mais les effectifs trop lourds et la précarité de son statut l'ont amené à se tourner vers la preschool Montessori.

\section{RÉSUMÉS}

À partir de recherches doctorales menées en 2013 et 2014, cet article a pour ambition d'analyser les processus qui ont conduit à une diffusion massive de l'enseignement préscolaire en Inde du sud. L'originalité du secteur préscolaire tient à la multiplicité des acteurs et à leurs enjeux différenciés. Plus particulièrement, il s'agit de comprendre les conséquences spatiales de l'engouement des familles et des institutions en faveur de la préscolarisation. Notre analyse porte ainsi un intérêt particulier au repositionnement de l'État, initiateur dès 1975 d'un projet en faveur de l'accueil des jeunes enfants des familles les plus modestes, et aujourd'hui confronté à la multiplicité des réponses.

Drawing on fieldwork research in 2013 and 2014, this article focuses on the processes which engender a widespread dissemination of preschooling in South India. The preschool sector presents originality, due to the wide variety of players involved and their challenges. It aims to understand the socio-spatial consequences of the success of preschool with families and institutions. The repositioning of the Government is interesting, it was initiator since 1975 of a preschooling project for children from the poorest families and now it facing a the multiplicity of responses.

\section{INDEX}

Mots-clés : Préscolarisation, Éducation, Classe Moyenne, Tamil Nadu, Inde

Keywords : Preschooling, Education, Middle classes, Tamil Nadu, India

Thèmes : Sur le Champ - Sur le Terrain

\section{AUTEUR}

\section{ÉMILIE PONCEAUD-GOREAU}

Emilie Ponceaud Goreau, emilie.ponceaud@etu.u-bordeaux3.fr, est doctorante en Géographie Humaine à l'Université Bordeaux Montaigne sous la direction de Yves Raibaud et Loraine Kennedy, rattachée à l'UMR ADESS (5185) et au Centre d'Etudes de l'Inde et de l'Asie du Sud (8564). Elle a publié récemment :

- Goreau A., Ponceaud-Goreau E., 2014. Le gendarme de Saint-Astier : les dispositifs spatiaux dédiés au maintien de l'ordre. EchoGéo [En ligne], 28 | 2014, mis en ligne le 8 juillet 2014. URL : http://echogeo.revues.org/13753 ; DOI : 10.4000/echogeo.13753

- Ponceaud-Goreau E., 2015 (à paraître). “Why go back to Pondicherry?” French Tamil Women in 
Return Migration. DESI: Indian Values in Diaspora: womanhood, $\mathrm{n}^{\circ} 3$, chap. 3.

- Ponceaud-Goreau E., 2015 (à paraître). Quelle identité donner à un espace original ? Le cas des cours d'écoles pré-primaires en Inde du sud. La cour de récréation : actes du colloque, Portet sur Garonne, Editions midi-pyrénéennes. 